\title{
Análises Biplot: conceitos, interpretações e aplicações
}

\author{
Biplot analysis: concepts, interpretations and uses
}

\author{
Raphael Rossi Silva ${ }^{\mathrm{I}}$ Giovani Benin ${ }^{\text {II }}$
}

- REVISÃo BIBLIOGRÁFICA -

\section{RESUMO}

A seleção e recomendação de genótipos superiores são dificultadas devido à ocorrência da interação genótipo e ambiente. Nesse contexto, as análises biplot têm sido cada vez. mais utilizadas na análise de dados agronômicos, com interações de natureza complexa. Entretanto, as particularidades existentes no gráfico biplot dificultam sua interpretação, podendo induzir o pesquisador a erros. Assim, este artigo de revisão discute a aplicabilidade e a interpretação gráfica dos modelos AMMI (Additive Main effects and Multiplicative Interaction) e GGE biplot (genotype main effects + genotype environment interaction) destas análises no gráfico biplot. Também, visa a desmistificar a necessidade de comparação entre ambas as metodologias. Discute-se quanto à escolha da metodologia mais adequada, levando em consideração a informação requerida $e$ os objetivos do pesquisador.

Palavras-chave: $A M M I$, GGE Biplot,interação genótipo e ambiente, adaptabilidade e estabilidade.

\section{ABSTRACT}

The genotype environment interaction (GE) influences on the selection and recommendation of cultivars. Biplot analysis has been increasingly used in data analysis of complex traits in agriculture. However, the peculiarities of biplot graphic could induce the researcher to errors on interpretation. Thus, this review argues the applicability and graphic interpretation of models AMMI (Additive Main Effects and Multiplicative Interaction) and GGE biplot (genotype main effects + genotype environment interaction). Moreover, also aims to explain that it is not adequate to compare both statistical methods. It is discussed the best methodology considering the information required and the research objectives.

Key words: $A M M I, G G E$ Biplot, genotype environment interaction, adaptability andstability.

\section{INTRODUÇÃO}

A expressão das características das plantas cultivadas está ligada ao controle genético, ao ambiente em que são cultivadas e à interação entre esses dois fatores (YAN\&KANG, 2003; MOHAMMAD\&AMRI, 2009). A resposta distinta dos genótipos em diferentes condições ambientais é denominada de interação genótipo e ambiente (GE), que reduz a correlação entre os valores fenotípicos e genotípicos e dificulta a seleção e recomendação de genótipos adaptados e estáveis (YAN\&HOLLAND, 2010).

Há diversas teorias para a avaliação da adaptabilidade e estabilidade, as quais diferem quanto aos seus conceitos e procedimentos biométricos de estimação. Dentre as metodologias mais recentes, têmse as análises AMMI (Additive Main effects and Multiplicative Interaction), que combina técnicas estatísticas para ajustar os efeitos principais (genótipos e ambientes) e os efeitos da GE (MANDEL, 1971; ZOBEL et al., 1988), cujo modelo é baseado em efeitos principais aditivos e interação multiplicativa; e o modelo

ICentro de Ciências Agrárias (CCA), Universidade Estadual de Londrina (UEL), Londrina, PR, Brasil.

"Universidade Tecnológica Federal do Paraná (UTFPR), Campus Pato Branco, Via do conhecimento, Km 01, 85503-390, Pato

Branco, PR, Brasil. E-mail: benin@utfpr.edu.br. Autor para correspondência. 
GGE biplot (genotype main effects + genotype environment interaction), proposto por YAN et al. (2000), que considera o efeito principal de genótipo mais a interação genótipo e ambiente. Ambas as análises, baseadas em gráficos biplot, representam graficamente uma matriz de dados.

A análise AMMI combina a análise de variância e a análise de componentes principais, para ajustar, respectivamente, os efeitos principais (genótipos e ambientes) e os efeitos da interação GE. Já a análise GGE agrupa o efeito aditivo de genótipo com o efeito multiplicativo da interação GE, e submete estes à análise de componentes principais (YAN et al., 2000).

Assim, o objetivo desta revisão bibliográfica é fornecer informações que auxiliem pesquisadores na escolha e utilização da metodologia mais adequada para a seleção de locais (ambientes) e recomendação de genótipos adaptados e estáveis.

\section{DESENVOLVIMENTO}

Teoria do biplot

O método biplot foi desenvolvido por GABRIEL (1971) para representar graficamente resultados de análise de componentes principais ou de decomposição de valores singulares, no qual o valor de cada elemento de uma tabela de dupla entrada pode ser visualizado pelo produto de vetores e pelo co-seno do ângulo entre dois vetores (YAN \& KANG, 2003). Quando duas matrizes apresentarem o mesmo número de linhas e colunas, é possível multiplicá-las. A nova matriz gerada da multiplicação das duas anteriores assume o mesmo número de linhas e colunas de ambas as matrizes (GABRIEL, 1971; YAN\&KANG, 2003). Como exemplo, tem-se a matriz G, denominada de "matriz linha" e a matriz E, denominada de "matriz coluna". A matriz $\mathrm{G}$ indica que há quatro genótipos e a matriz $\mathrm{E}$, que há três ambientes.

$$
\text { Matriz } G=\left[\begin{array}{cc}
5 & 2 \\
-2 & 3 \\
2 & 4 \\
1 & 0
\end{array}\right] \text { e Matriz } E=\left[\begin{array}{ccc}
1 & 4 & -2 \\
2 & 6 & 1
\end{array}\right]
$$

A matriz $\mathrm{G}$ possui duas colunas e três linhas e a E possui duas linhas e três colunas, sendo possível multiplicá-las. A matriz produto, denominada de Y, tem quatro linhas e três colunas. A regra da multiplicação de matrizes é que a matriz Y seja a soma do produto entre cada elemento da linha na matriz $\mathrm{G}$ e cada elemento da coluna na matriz E. Assim, os elementos de Y são calculados conforme segue:

(1) $\mathrm{Y}_{11}=((\mathrm{G} 11 * \mathrm{E} 11)+(\mathrm{G} 12 * \mathrm{E} 21))=((5 * 1)+(2 * 2))=9$

(2) $\left.\mathrm{Y}_{31}=((\mathrm{G} 31 * \mathrm{E} 11)+(\mathrm{G} 32 * \mathrm{E} 21))=(2 * 1)+(4 * 2)\right)=10$
A multiplicação das matrizes $\mathrm{Ge}$ E possibilita a obtenção de 12 elementos, os quais podem ser observados na matriz Y, a qual, segundo GABRIEL (1971), pode ser representada em um gráfico biplot bidimensional.

$$
\mathrm{Y}=\left[\begin{array}{ccc}
9 & 32 & -12 \\
4 & 10 & 1 \\
10 & 30 & -8 \\
1 & 4 & -2
\end{array}\right]
$$

A plotagem dos valores $x$ e $y$ das matrizes $\mathrm{G}$ e $\mathrm{E}$ originam o gráfico biplot, que são representados por pontos (Figura 1). A distância entre a origem do biplot $(o)$ e o ponto $\mathrm{E}_{2}$, por exemplo, é chamado vetor de $E_{2}$. $O$ elemento $Y_{x}$ pode ser obtido através do produto do vetor de $\mathrm{G}_{\mathrm{x}}$ pelo co-seno do ângulo entre $E_{x}$ e $G_{x}$ e pelo vetor de $E_{x}$, através da seguinte fórmula:

(3) $Y_{12}=\overline{o G 1} \cos \alpha \overline{o E 1}$

Em que, $\overline{o G 1}=\sqrt{x 1^{2}+y 1^{2}} ; \overline{o E 1}=\sqrt{x 2^{2}+y 2^{2}}$; $\alpha 12+\beta=\operatorname{arctg}(y 2 / x 2) ; \beta=\operatorname{arctg}(y 1 / x 1) ; \alpha 12=((\alpha+\beta)-\beta)$.

Os valores de $\overline{o G i}$ e $\overline{o E i}$ nunca podem ser negativos, porém o valor do co-seno do ângulo pode ser positivo ou negativo, a depender do ângulo. $\mathrm{O}$ elemento $\mathrm{Y}_{12}$, por exemplo, pode ser: 0 se o ângulo for $90^{\circ}$, negativo se for maior que $90^{\circ}$ e positivo se o ângulo for menor que $90^{\circ}$.

As figuras 1, $2 \mathrm{e} 3$ apresentam a teoria biplot sem a aplicação dos modelos GGE ou AMMI. A partir do momento que o gráfico passa a representar uma ou outra metodologia, não se pode generalizar as inferências (YAN \& KANG, 2003). No modelo GGE, primeiro está embutido o efeito principal do genótipo somado ao da GE, enquanto que no modelo AMMI é possível observar o efeito da GE e do ambiente.

A figura $2 \mathrm{a}$, em que $G$ e $E$ designam genótipos e ambientes, respectivamente, ilustra a comparação entre os pontos $\mathrm{G}_{2}$ e $\mathrm{G}_{4}$. Para tal, traça-se uma linha conectando ambos os genótipos e uma linha perpendicular à linha conectora dos genótipos, passando pela origem do biplot (YAN\&TINKER, 2006). $\mathrm{O}$ ponto $\mathrm{E}_{3}$ ficou do mesmo lado que $\mathrm{G}_{2}$, o que possibilita inferir que $\mathrm{G}_{2}$ é o melhor adaptado a $\mathrm{E}_{3}$, comparativamente $\mathrm{a}_{4}$. Na figura $2 \mathrm{~b}$, que compara dois ambientes, apenas $\mathrm{G}_{2}$ é adaptado a $\mathrm{E}_{3}$, enquanto os demais genótipos são adaptados a $\mathrm{E}_{1}$.

A visualização do melhor desempenho de determinado genótipo em cada ambiente se dá interligando todos os genótipos que estão nos pontos extremos do gráfico e suas respectivas linhas perpendiculares, formando um polígono (YAN\&KANG, 2003; YAN\&TINKER, 2006). Esse polígono apresenta a melhor cultivar para cada ambiente e divide os ambientes em grupos (ALWALA 


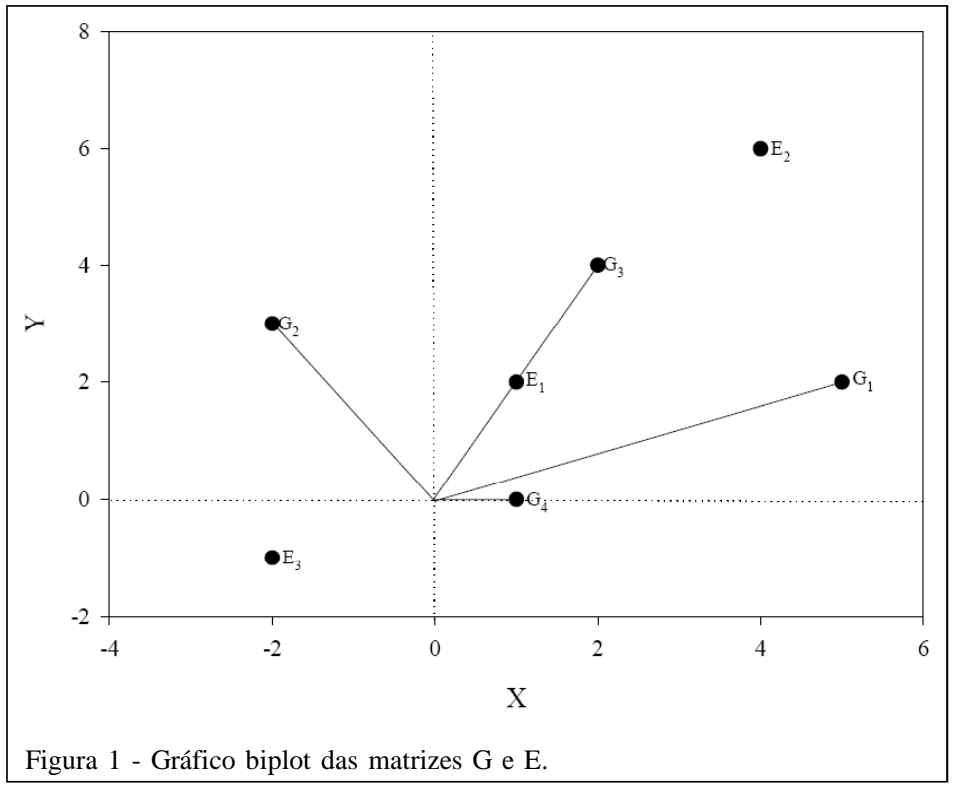

et al., 2010). Na Figura $2 c$, observa-se que $\mathrm{G}_{2}$ é adaptado ao Setor 4 (E3). Os genótipos localizados dentro de um mesmo setor são os mais adaptados àqueles ambientes. Assim, ao Setor 3, adaptaram-se $\mathrm{G}_{3}, \mathrm{G}_{1}$ e $\mathrm{G}_{4}$ e, no Setor 4 , apenas $\mathrm{G}_{2}$.

Quando diferentes cultivares estão adaptadas a diferentes grupos de ambientes e a variação entre grupos é maior do que dentro do grupo, tem-se a formação de um mega-ambiente (YAN \& KANG, 2003). A definição de mega-ambientes e a relação entre os ambientes auxiliam fitotecnistas e melhoristas de plantas na identificação de genótipos que possuam adaptação ampla ou específica a determinados ambientes ou grupos de ambientes (TRETHOWAN, et al., 2001).

$\mathrm{Na}$ análise GGE biplot, quando se estuda mega-ambientes, a média no gráfico não está relacionada à média geral, mas sim à média do mega-ambiente (YAN \& TINKER, 2006). Portanto, a média de cada cultivar é comparada dentro do mega-ambiente, não sendo possível comparar $\mathrm{G}_{1}$ e $\mathrm{G}_{4}$ com $\mathrm{G}_{2}$, pois este último está alocado no Setor 4 (Figura 2c). Esta abordagem auxilia na identificação de genótipos que possuam adaptação ampla ou específica a determinados ambientes ou grupos de ambientes (TRETHOWAN et al., 2001).

O desempenho de um dado genótipo ou ambiente é observado em relação ao eixo $x$, em que quanto mais à direita do centro (o) do biplot maior é o desempenho e, quanto mais à esquerda, menor o desempenho. Genótipos ou ambientes localizados à esquerda e à direita do centro do biplot apresentam desempenho inferior e superior à média geral, respectivamente. A estabilidade é visualizada no eixo $y$, em que, quanto mais distante do centro $(o)$ do biplot, menor é a estabilidade (GABRIEL, 1971; YAN\&KANG, 2003). Portanto, os genótipos $G_{1}$ e $G_{2}$ apresentam o maior e menor rendimento de grãos, respectivamente, enquanto que $G_{4}$ e $G_{3}$ são os de maior e menor estabilidade, respectivamente (Figura 3a).

Ainda na figura 3a, é possível identificar as associações de cada genótipo (G) nas diferentes condições ambientais (E), em que os genótipos que se localizam no mesmo quadrante dos ambientes estão positivamente associados àqueles ambientes. Assim, $\mathrm{G}_{1}, \mathrm{G}_{3}$ e $\mathrm{G}_{4}$ apresentam associação positiva com $\mathrm{E}_{1} \mathrm{e} \mathrm{E}_{2}$. Entretanto, também é necessário observar a distância do genótipo em relação ao marcador do ambiente, pois $\mathrm{G}_{4}$ está fortemente associado (adaptado) a $\mathrm{E}_{1}$, enquanto $\mathrm{G}_{3}$ apresenta adaptação tanto a $\mathrm{E}_{2}$ quanto $\mathrm{E}$.

Quando se analisa o co-seno do ângulo entre genótipo e ambiente, há maior exatidão na identificação das associações positivas, pois deixa de ser visual e analisa-se matematicamente. A associação entre $\mathrm{G}_{2} \mathrm{e} \mathrm{E}_{3}$ é o co-seno da soma dos ângulos a2 e b2 (0.12), o qual indica associação positiva de baixa magnitude; a associação entre $\mathrm{G}_{1}$ e $\mathrm{E}_{2}$ é o co-seno da subtração dos ângulos a1 e b1(0.82), indicando que $\mathrm{G}_{1}$ apresenta associação positiva de elevada magnitude $\operatorname{com~}_{2}$ (Figura $3 b$ ). As demais associações, tanto entre genótipos quanto entre ambientes, seguem o mesmo raciocínio. 


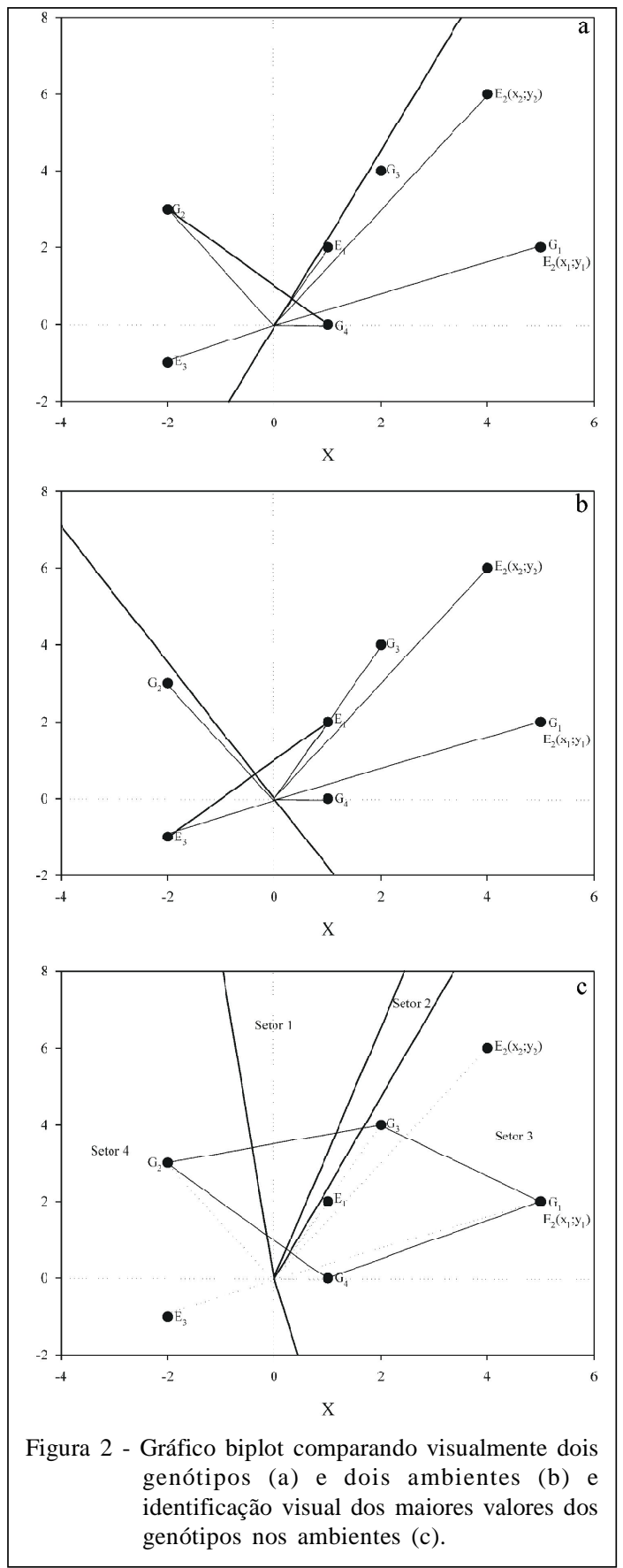

\section{Modelo GGE biplot}

Na metodologia GGE, o co-seno do ângulo entre dois ambientes corresponde à correlação genética entre eles. Outros tipos de biplots não apresentam essa propriedade (YAN et al., 2007), o que torna essa metodologia mais eficiente em relação a outras técnicas baseadas em biplot (YAN, 2011). Tal metodologia é descrita da seguinte maneira:

(4) $Y_{i j}=\mu+\alpha_{i}+\beta_{j}+\Phi_{i j}$
Em que, $\mathrm{Y}_{\mathrm{ij}}$ é o desempenho esperado do genótipo $i$ no ambiente $j$; $\mu$ é a média geral das observações; $\alpha_{i}$ é o efeito principal do genótipo $i ; \beta_{j \text { é }}$ o efeito principal do ambiente $j ; \Phi_{i j}$ é a interação entre o genótipo $i$ e o ambiente $j$.

Logo, nessa análise, a variação fenotípica é resultante do efeito genotípico $\left(\alpha_{i}\right)$, do ambiente $\left(\beta_{j}\right)$ e da interação entre genótipo e ambiente $\left(\Phi_{i}\right)$ (YAN\&KANG, 2003). Nesta metodologia, apenas o efeito principal de genótipo e da GE são importantes, devendo ser considerados concomitantemente. 


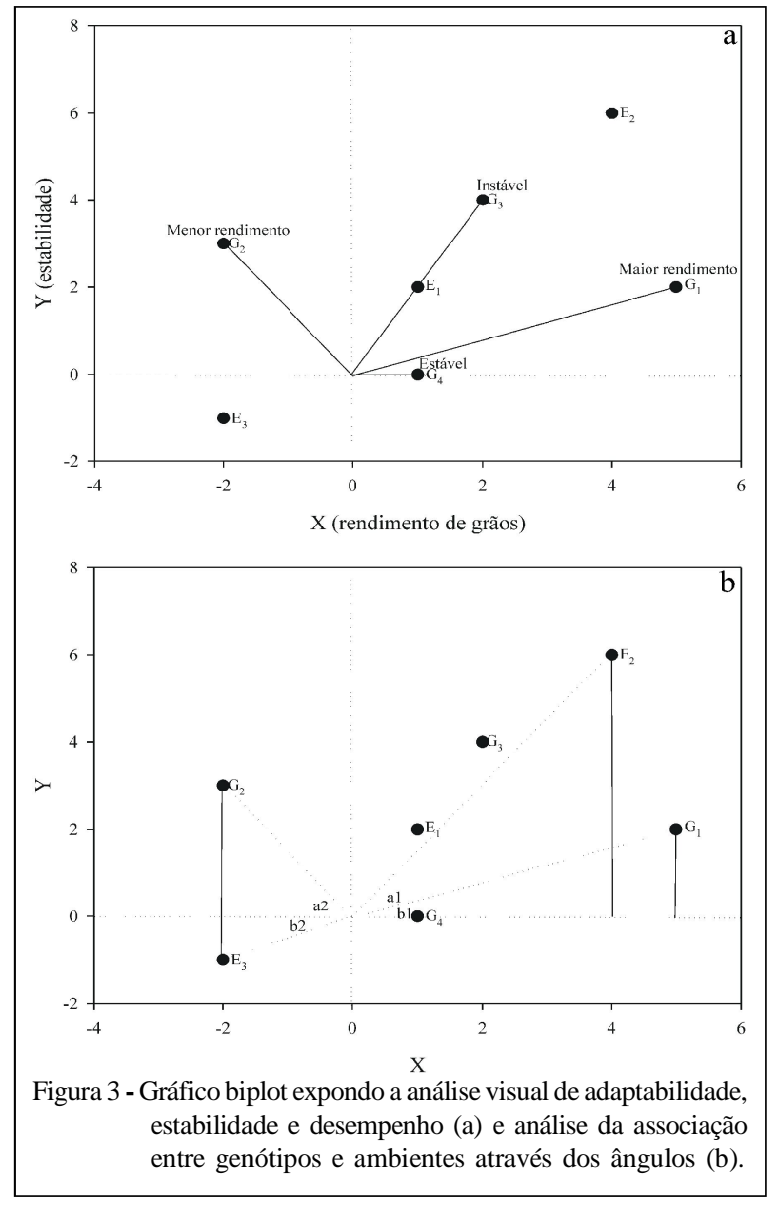

Considera-se que o efeito principal do ambiente não é relevante na seleção de cultivares. Isso se deve ao fato de que, na seleção de cultivares e formação de megaambientes, a capacidade adaptativa dos genótipos é mais importante em relação às condições agroclimáticas (CAMARGO-BUITRAGO et al., 2011). O modelo GGE biplot não separa $\mathrm{G}$ da GE, mantendo-os juntos em dois termos multiplicativos, que podem ser visualizado na seguinte equação.

$$
\text { (5) } Y_{i j}-\mu-\beta_{j}=g_{i 1} e_{i 1}+g_{i 2} e_{i 2}+\varepsilon_{i j}
$$

Em que, $\mathrm{Y}_{\mathrm{ij}}$ é o rendimento esperado do genótipo $i$ no ambiente $j ; \mu$ é a média geral das observações; $\alpha_{i}$ é o efeito principal do genótipo $i ; \beta_{j}$ é o efeito principal do ambiente $j ; \mathrm{g}_{\mathrm{i} 1} \mathrm{e} \mathrm{e}_{\mathrm{i} 1}$ são denominados escores principais do genótipo $i$ e ambiente $j$, respectivamente; $\mathrm{g}_{\mathrm{i} 2}$ e $\mathrm{e}_{\mathrm{i} 2}$ são os escores secundários para o genótipo $i$ e ambiente $j$, respectivamente; $\varepsilon_{i j}$ é o resíduo não explicado por ambos os efeitos.

Assim sendo, a construção do gráfico biplot no modelo GGE se dá através da dispersão simples de $\mathrm{g}_{\mathrm{i} 1}$ e $\mathrm{g}_{\mathrm{i} 2}$ para genótipos e $\mathrm{e}_{\mathrm{i} 1}$ e $\mathrm{e}_{\mathrm{i} 2}$ para ambientes. $\mathrm{O}$ escore primário é obtido através da decomposição do valor singular (SVD) nas três matrizes oriundas de uma tabela que apresenta os valores de genótipos e ambientes. A SVD é importante na análise biplot, pois permite computar os componentes principais e, também, fornece uma medida da variabilidade capturada por cada um dos componentes (YAN, 2002; YAN \& TINKER, 2006).

YAN \& TINKER (2006) expuseram a decomposição de valor singular (SVD) através da seguinte fórmula: $\mathrm{P}_{\mathrm{mn}}=\mathrm{G}_{\mathrm{m}, \mathrm{r}} \mathrm{G}_{\mathrm{r}, \mathrm{r}} \mathrm{E}_{\mathrm{n}, \mathrm{r}}^{\mathrm{T}}(\mathrm{r} \leq \min (\mathrm{m}, \mathrm{n}))$. A matriz $\mathrm{G}$ tem $m$ linhas e $r$ colunas, caracterizando $m$ genótipos. A matriz $\mathrm{E}$ tem $r$ linhas e $n$ colunas, que caracterizam os ambientes. AMatriz Lé a matriz diagonal contendo $r$ valores singulares. Em suma, a SVD decompõe os valores da tabela $(\mathrm{P})$ de dados em $r$ componentes principais, cada um contendo o vetor genótipo $\left(\xi_{\mathrm{i}}\right)$, o vetor ambiente $\left(\eta_{\mathrm{j}}\right)$ e os valores singulares $(\lambda)$, que compõem a seguinte equação:

(6) $\mathrm{P}_{\mathrm{ij}}=\sum_{1}^{\mathrm{r}}=1 \xi_{\mathrm{i} 1} \lambda_{1} \eta_{1 \mathrm{j}}\left(\lambda_{1} \geq \lambda_{1+1}\right)$

Em que $r$ é a classificação na tabela nos dois sentidos (genótipo e ambiente), ou seja, o número de componentes principais necessários para representar 
$\mathrm{P}, \operatorname{com} \mathrm{r} \leq \min (\mathrm{m}, \mathrm{n})$. Se $\mathrm{r}<\mathrm{m}$ é indicativo de que há associação entre os genótipos e se $r<n$ há associações entre os ambientes.

No entanto, os autovetores PC1 (componente principal 1) e PC2 (componente principal 2) não podem ser plotados diretamente antes dos valores singulares serem particionados em genótipos e ambientes. A partição é expressa pela seguinte fórmula:

$$
\text { (7) } P_{\mathrm{ij}}=\sum_{\mathrm{i}=1}^{\mathrm{r}} \xi_{\mathrm{il}} \eta_{\mathrm{ij}}=\sum_{\mathrm{i}=1}^{\mathrm{r}}\left(\xi_{\mathrm{il}} \lambda_{1}\right)\left(\lambda_{1}^{1-\mathrm{f}} \eta_{\mathrm{ij}}\right)
$$

Em que, $f$ é o fator de partição, que, teoricamente, pode ser qualquer valor entre 0 e 1 . Entretanto, YAN \& KANG (2003) ressaltaram que diferentes valores de $f$, são capazes de alterar a visualização das inter-relações entre genótipos e, também, entre os ambientes.

Portanto, quando $f_{s l}=1$ os valores singulares são particionados totalmente nos autovetores de genótipo, sendo que os comprimentos dos vetores podem variar e estão associados aos valores singulares que representam a unidade original dos dados. Por outro lado, quando $f_{s l}=0$ os valores singulares são particionados nos autovetores de ambiente, no qual o tamanho dos vetores é semelhante, ou seja, os ambientes têm peso igual na GxE. YAN \& TINKER (2006) explicam que o modelo focado no ambiente é importante, pois a correlação pode ser obtida através do co-seno dos ângulos, indicando a capacidade de discriminação dos ambientes. Quando o interesse for visualizar a similaridade entre os genótipos, o modelo focado no genótipo é a forma apropriada para a visualização dos dados (YAN\&TINKER, 2006).

\section{Metodologia AMMI}

O gráfico biplot do modelo AMMI (additive main effects and multiplicative interaction analysis) é obtido através da análise de variância e da decomposição singular dos valores observados. $\mathrm{Na}$ primeira etapa, a ANOVAé aplicada à matriz de médias, composta pelos efeitos principais da parte aditiva (média geral, efeitos genotípicos e ambientais), resultando em um resíduo de não aditividade, que compõe a parte multiplicativa do modelo. Na segunda fase, é analisada a decomposição dos valores singulares da matriz de interações. No modelo, unem-se os termos aditivos dos efeitos principais e os termos multiplicativos dos efeitos da interação.

O modelo da metodologia AMMI pode ser demonstrado por:

$$
\text { (8) } Y_{i j}=\mu+g_{i}+\alpha_{j}+\sum_{k=1}^{n} \lambda_{k} \gamma_{i k} \alpha_{j k}+\rho_{i j} \varepsilon_{i j}
$$

Em que, $\mathrm{Y}_{\mathrm{ij}}$ é a resposta média do genótipo $i$ (i $=1,2, \ldots, \mathrm{G}$ genótipos) no ambiente $j(\mathrm{j}=1,2, \ldots, \mathrm{A}$ ambientes); $\mu$ é a média geral dos ensaios; $\mathrm{g}_{\mathrm{i}}$ é o efeito fixo do genótipo $i ; \alpha_{j}$ é o efeito fixo do ambiente $j ; \lambda_{k}$ é o k-ésimo valor singular (escalar) da matriz de interações original (denotada por GE); $\gamma_{i k}$ é o elemento correspondente ao i-ésimo genótipo, no k-ésimo vetor singular coluna da matriz GE; $\alpha_{j k}$ é o elemento correspondente ao j-ésimo ambiente, no k-ésimo vetor singular linha da matriz GE; $\rho_{i j}$ é o resíduo associado ao termo (ga)ij da interação clássica do genótipo $\boldsymbol{i}$ com o ambiente $j ; \varepsilon_{i j}$ é o erro experimental médio.

Na análise AMMI, é indispensável identificar o número de eixos que serão empregados na análise, enquanto que, na GGE, são apenas dois eixos. Um dos procedimentos adotados na definição do número de eixos para explicar o padrão relacionado à interação consiste em determinar graus de liberdade associados à parcela da soma de quadrados, devido à interação relacionada a cada modelo da análise AMMI (AMMI0, AMMI1, AMMI2, ..., AMMIn). Obtém-se então o quadrado médio correspondente a cada parcela (ou modelo) e, na sequencia, é obtido um teste F, avaliando-se a significância de cada componente em relação ao quadrado médio do erro.

A significância do teste $\mathrm{F}$ determina $\mathrm{o}$ modelo a ser empregado nas análises. Sobre o modelo AMMI selecionado, determinam-se os escores dos níveis de cada fator pela decomposição dos valores singulares, que permitem explorar o efeito positivo da interação. Entretanto, como há existência de maior padrão nos dois primeiros eixos, a representação gráfica AMMI2 é a mais utilizada, pois a principal finalidade da análise AMMI é selecionar modelos que expliquem o padrão relacionado à interação, desprezando-se os ruídos (CROSSA, 1990). A presença de maior ruído em modelos após AMMI2 ocorre, pois, a cada modelo selecionado, desde o AMMI0, é determinado graus de liberdade associados à soma de quadrados da GE, em que o modelo a ser adotado avalia a significância de cada componente em relação ao quadrado médio do erro (ruídos) (LAVORANTI et al., 2004).

\section{GGE biplot versusAMMI}

O modelo GGE biplot apresenta o efeito de genótipo como um efeito multiplicativo da GE. Como, em geral, o PC1 está correlacionado com o efeito do genótipo, é possível alta precisão na visualização gráfica do escore $\mathrm{PC} 1$ quanto ao efeito genotípico. YAN et al. (2000) acreditam que esta proporcionalidade da resposta de genótipos seja mais lógica e biologicamente plausível, pois há correlação quase perfeita entre o rendimento de grãos e o PC1 (NETO- FRITSCHE et al., 2010; SINEBO et al., 2010). Por outro lado, no modelo AMMI2, tem-se observado correlação negativa entre IPC1 e o rendimento (KVITSCHAL et al., 2009). Entretanto, no modelo AMMI1, há correlação quase 
perfeita com o rendimento de grãos, assim como na GGE biplot, pois é um modelo baseado no IPC1 versus rendimento de grãos.

O modelo AMMI possui maior acurácia na visualização do padrão em experimentos, quando comparado ao GGE biplot (GAUCH, 2006). Entretanto, o modelo GGE é mais vantajoso em relação ao modelo AMMI1 (IPC1 x rendimento), pois é capaz de explicar uma maior porcentagem da GE em relação ao gráfico AMMI1 (YAN et al., 2007). Nesse sentido, enquanto a análise GGE captou 77,3\% do padrão, o modelo AMMI1 captou $64.6 \%$, segundo SAMONTE et al. (2005) e GAUCH et al. (2008). O modelo AMMI2, normalmente, apresenta maior porcentagem de explicação do padrão, quando comparado ao GGE biplot, sendo indicado como o melhor modelo da análise AMMI (GAUCH et al., 2008; BALLESTRE et al., 2009). Entretanto, nem sempre a maior captação de porcentagem de explicação representa maior precisão na seleção de genótipos superiores, isso porque pode haver ruídos na porcentagem de explicação.

O modelo AMMI1 é menos adequado em relação ao GGE para a definição de mega-ambientes, pois, no modelo AMMI1, os ambientes são apresentados apenas na abscissa e os genótipos não são apresentados por pontos, mas por linhas retas (YAN et al., 2007). GAUCH et al. (2008) contestaram tal comparação afirmando que o modelo AMMI1 possui geometria mais simples e informativa e que capta menos ruídos do que o GGE. Além disso, Yan et al. (2011) relataram que, normalmente, o modelo GGE biplot é capaz de reter maior explicação da G+GE. Contudo, estudos recentes demonstram que ambas as metodologias, quando utilizadas adequadamente, são eficientes na definição de mega-ambientes (CAMARGO-BUITRAGO et al., 2011; YAN, 2011). Comparando as metodologias AMMI1 e GGE Biplot na formação de mega-ambientes, CAMARGO-BUITRAGO et al. (2011) relataram 95,2\% de coincidência nos resultados entre as metodologias.

GAUCH (2006) critica o modelo GGE biplot, pois esse não separa o efeito do genótipo $(\mathrm{G})$ da interação genótipo ambiente (GE), tal como a análise AMMI. Entretanto, YAN et al. (2007) discordam, pois melhoristas e fitotecnistas têm interesse em selecionar plantas com base no $\mathrm{G}$ e na GE, simultaneamente. Assim, o efeito principal do ambiente não tem importância quando se realizam testes de cultivares, pois este efeito é irrelevante na identificação de genótipos superiores (YAN et al., 2003). Segundo estes autores, embora o desempenho da cultivar seja o efeito combinado do genótipo, ambiente e da GE, apenas G e GE são relevantes e devem ser considerados simultaneamente na avaliação da adaptabilidade e estabilidade de genótipos.
A única diferença entre as metodologias GGE e AMMI é que a primeira analisa diretamente o efeito de G+GE, enquanto que a AMMI separa G da GE e depois os juntam para formar o gráfico (YAN et al., 2008). Esses autores alegam que essa separação não é capaz de conferir superioridade à análise AMMI. Nesse contexto, YAN et al. (2007) argumentam que a separação de G da GE pode acarretar em equívocos na seleção de genótipos, pois a GE é apenas um dos componentes da adaptação específica. Portanto, um genótipo pode interagir negativamente com determinado ambiente, mas, ainda assim, apresentar alto rendimento nessa condição ambiental.

Nesse sentido, estudos indicam que a análise GGE biplot é de fácil compreensão e pode contribuir efetivamente na identificação de megaambientes (YAN \& RAJCAN, 2002), seleção de cultivares de adaptabilidade ampla ou específica (DEHGHANI et al., 2006; KANG et al., 2006). Além disso, tem sido eficaz para identificar genótipos superiores e estáveis, principalmente, quando testados em grande número de ambientes (ALWALA et al., 2010).

Assim, o modelo GGE biplot é mais indicado para a identificação de mega-ambientes, seleção de ambientes representativos e discriminativos e indicação de cultivares mais adaptadas e estáveis a ambientes específicos (YAN et al., 2007; GAUCH et al., 2008; ASFAW et al., 2009; YAN, 2010). Por outro lado, a análise AMMI pode ser utilizada com eficiência na identificação de condições ambientais superiores para a exploração agrícola (seleção de locais de cultivo) e genótipos de superior desempenho médio (GAUCH et al.; 2008; YAN, 2010).

\section{CONCLUSÃO}

A utilização conjunta das metodologias AMMI e GGE Biplot torna mais eficaz a exploração da interação genótipo versus ambiente, com subsídios à seleção de genótipos e ambientes superiores.

O modelo AMMI1 pode ser utilizado com segurança quando se deseja selecionar, principalmente, os melhores locais de cultivo e genótipos de superior desempenho médio.

O modelo GGE explora com mais eficácia a interação genótipo e ambiente, possibilitando maior acurácia à identificação de mega-ambientes e a seleção de genótipos estáveis e adaptados a ambientes específicos e a mega-ambientes.

O maior mérito da metodologia GGE biplot é quando um grande número de genótipos é testado em diversas condições ambientais e quando o padrão da interação genótipo e ambiente é de maior complexidade. 


\section{REFERÊNCIAS}

ALWALA, S. et al. A comprehensive comparison between Eberhart and Russel joint regression and GGE biplot analyses to identify stable and high yielding maize hybrids. Field Crops Research, v.119, n.2-3, p.225-230, 2010.

ASFAW, A. et al. AMMI and GGE Biplot anlysis for matching varieties onto soybean production environments in Ethiopia. Scientific Research and Essay, v.4, n.11, p.1322-1330, 2009. Disponível em: <www.academicjournals.org/sre/PDF/pdf2009/ Nov/Asfaw\%20et\%20al.pdf>. Acesso em:17 out. 2010.

BALESTRE, M. et al. Yield stability and adaptability of maize hybrids based on GGE biplot analysis characteristics. Crop breeding and applied biotechnology, v.9, n3, p.219-228, 2009. Disponível em: <http://www.sbmp.org.br/cbab/siscbab/ uploads/c8eb9791-1c9f-e792.pdf>. Acesso em: 05 nov. 2010.

CAMARGO-BUITRAGO, I. et al. Identificación de megaambientes para potenciar el uso de genótipos superiores de arroz en Panamá. Pesquisa Agropecuária Brasileira, v.46, n.9, p.1601-1069, 2011. Disponível em: <http://www.scielo.br/ pdf/pab/v46n9/46n09a13.pdf>. Acesso em: 24 dez. 2011. doi:10.1590/S0100-204X2011000900013.

CROSSA, J. Statistical analyses of multilocation trials. Advances in Agronomy, v.44, n.1, p.55-85, 1990. Disponível em: <http:/ /www.sciencedirect.com/science/article/pii/S0065211308608184>. Acesso em: 20 jul. 2010. doi: 10.1016/S0065-2113(08)60818-4.

DEHGHANI, H. et al. Biplot analysisof genotype by environment interaction for barley yield in Iran. Agronomy Journal, v.98, n.2, p.388-393, 2006. Disponível em: <https:/ /www.crops.org/publications/aj/articles/98/2/388>. Acesso em: 09 dez. 2011. doi: 10.2134/agronj2004.0310.

GABRIEL, K.R. The biplot graphic display of matrices with application to principal componente analysis. Biometrika, v.58, n.3, p.453-467, 1971. Disponível em: <http:// biomet.oxfordjournals.org/content/58/3/453.abstract>. Acesso em: 20 jul. 2010. doi: 10.1093/biomet/58.3.453.

GAUCH, H.G. et al. Statistical analysis of yield trials by AMMI and GGE: further considerations. Crop science, v.48, n3, p.866-889, 2008. Disponível em: <https://www.crops.org/ publications/cs/articles/48/3/866>. Acesso em: 05 maio, 2010. doi: 10.2135/cropsci2007.09.0513.

GAUCH, H.G. Statistical analysis of yield trials by AMMI and GGE. Crop Science, v.46, n.4, p.1488-1500, 2006. Disponível em: 〈https://www.crops.org/publications/cs/articles/46/4/1488>. Acesso em: 05 maio, 2010. doi: 10.2135/cropsci2005.07-0193.

KANG, M.S. et al. Adaptabilityand stability of bean cultivars as determined via yield-stabilitystatistic and GGE Biplot analysis. Journal of Crop Improvement, v.15, n.1, p.97-120, 2006. Disponível em: <http://www.tandfonline.com/doi/abs/10.1300/ J411v15n01_08>. Acesso em: 09 dez. 2011. doi: 10.1300/ J411v15n01_08.

KVITSCHAL, M.V. et al. Comparison of methods for phenotypic stability analysis of Cassava (Manihot esculenta Crantz) genotypes for yield and storage root dry matter content. Brazilian Archives of Biology and Technology, v.52, n.1, p.163-175, 2009. Disponível em: <http://www.scielo.br/pdf/ babt/v52n1/a22v52n1.pdf>. Acesso em: 05 maio, 2010. doi: 10.1590/S1516-89132009000100021.

LAVORANTI, O.J. et al. Modelagem AMMI para estudos de interação em modelos estatísticos de efeitos fixos. Colombo: EMBRAPA Florestas, 2004. 7p. (Comunicado Técnico, 124).

MANDEL, J. A new analysis of variance model fornon-additive data. Technometrics, v.13, p.1-18, 1971. Disponível em: <http://www.jstor.org/pss/1267072〉. Acesso em: 01 out. 2010.

MOHAMMADI, R.; AMRI, A. Analysis of genotype $\mathrm{x}$ environment interactions for grain yield in durum wheat. Crop Science, v.49, n.4, p.1177-1186, 2009. Disponível em: <https://www.crops.org/publications/cs/articles/49/4/1177>. Acesso em: 10 maio, 2010. doi: 10.2135/cropsci2008.09.0537.

NETO-FRITSCHE, R. et al. Factor analysis and SREG GGE biplot for the genotype $\mathrm{x}$ environment interaction stratification in maize. Ciência Rural, v.40, n.5, p.1043-1048, 2010. Disponível em: <http://www.scielo.br/pdf/cr/v40n5/a576cr2396.pdf>. Acesso em: 10 out. 2010 . doi: 10.1590/S0103-84782010000500007.

SAMONTE, S.O.P.B. et al. Targeting cultivars onto rice growing environments using AMMI and SREG GGE biplot analyses. Crop Science, v.45, n.6, p.2414-2424, 2005. Disponível em: <https://www.crops.org/publications/cs/articles/45/6/2414>. Acesso em: 05 maio, 2010. doi: 10.2135/cropsci2004.0627.

SINEBO, W. et al. Biplot analysis of grain yield barley grown under differing management levels in years of contrasting season-end drought. Journal of Plant Breeding and Crop Science, v.2, n.6, p.152-164, 2010. Disponível em: <http:// www.academicjournals.org/jpbcs/PDF/pdf\%202010/June/ Sinebo\%20et\%20al.pdf>. Acesso em: 10 jul. 2010.

TRETHOWAN, R.M. et al. Relationships among bread wheat international yield testing locations in dry areas. Crop Science, v.41, n.5, p.1461-1469, 2001. Disponível em: <https:// www.crops.org/publications/cs/articles/41/5/1461>. Acesso em: 10 maio, 2010. doi: $10.2135 /$ cropsci2001.4151461x.

YAN, W. Optimal use of biplots in analysis of multi-location variety test data. Acta Agronomica Sinica, v.36, n.11, p.18051819, 2010. Disponível em: <http://211.155.251.148:8080/ zwxb/CN/article/showZhaiYao.do?id=4677\#>. Acesso em: 20 maio, 2011. doi: 10.3724/SP.J.1006.2010.01805.

YAN, W. et al. Cultivar evaluation and mega-environment investigation based on the GGE Biplot. Crop Science, v.40, n.3, p.597-605, 2000. Disponível em: <https://www.crops.org/ publications/cs/articles/40/3/597>. Acesso em: 20 maio, 2010. doi: $10.2135 /$ cropsci2000.403597x.

YAN, W. et al. GGE Biplots vs. AMMI analysis of genotypeby-environment data. Crop science, v.47, n.2, p.643-655, 2007. Disponível em: <https://www.crops.org/publications/cs/ articles/47/2/643>. Acesso em: 10 maio, 2010. doi: 10.2135/ cropsci2006.06.0374.

YAN, W. GGE biplot vs. AMMI graphs for genotype-byenvironment data analysis. Journal of the Indian Society of Agricultural Statistics, v.65, n.2, p.181-193, 2011. Disponível em: <http://isas.org.in/jisas/jsp/volume/vol65/issue2/ 06-Weikai\%20Yan.pdf>. Acesso em: 09 dez. 2011. 
YAN, W. Singular-value partitioning in biplot analysis of multienvironment trial data. Agronomy Journal, v.94, n.5, p.990-996, 2002.

YAN, W.; HOLLAND, J.B. A heritability-adjusted GGE biplot for test environment evaluation. Euphytica v.171, n.3, p.355369, 2010. Disponível em: <http://www.springerlink.com/ content/p816144m1574x358/fulltext.pdf>. Acesso em: 20 ago. 2010. doi: 10.1007/s10681-009-0030-5.

YAN, W.; KANG, M.S. GGE biplot analysis: a graphical tool for breeders, geneticists, and agronomists. Flórida: Boca Raton, 2003. 286p.

YAN, W.K.; RAJCAN, I. Biplot analysis of test sites and trait relations of soybean in Ontario. Crop Science, v.42, n.1, p.11-20, 2002. Disponível em: <https://www.soils.org/ publications/cs/articles/42/1/11>. Acesso em: 09 dez. 2011. doi: $10.2135 /$ cropsci2002.1100.

YAN, W; TINKER, A. Biplot analysis of multi environment trial data: principles and applications. Canadian Journal of Plant Science, v.86, n.3, p.623-645, 2006. Disponível em: <http://pubs.aic.ca/doi/abs/10.4141/P05-169>. Acesso em: 05 maio, 2010. doi: 10.4141/P05-169.

ZOBEL, R.W. et al. Statistical analysis of a yield trial. Agronomy Journal, v.80, n.3, p.388-393, 1988. Disponível em: <https://www.agronomy.org/publications/aj/abstracts/80/3/ AJ0800030388 ?access $=0 \& v i e w=p d f>$. Acesso em: $24 \mathrm{dez}$. 2011. doi: 10.2134/agronj1988.00021962008000030002x. 